\title{
Methods for assessing public health vulnerability to global climate change
}

\author{
Jonathan A. Patz ${ }^{1}$, John M. Balbus ${ }^{2}$ \\ ${ }^{1}$ Department of Molecular Microbiology \& Immunology, Johns Hopkins School of Hygiene and Public Health, Baltimore, \\ Maryland 21205-2179, USA \\ ${ }^{2}$ Department of Occupational \& Environmental Medicine, George Washington University, School of Medicine, \\ Washington, DC, USA
}

\begin{abstract}
Assessment of the human health risk posed by global climate change presents a new challenge to public health professionals. In contrast to conventional toxicological risk assessment, the health risk assessment related to global climate change must analyze stressors that consist of complex interrelated climate factors and risks that are mediated through intermediate species in varying ecosystems. A framework for ecologically based human health risk assessment helps distinguish the concepts of global climate change risk assessment from conventional risk assessment. Specific methods for linking climate variables with human disease include historical analysis of climate and disease data and the development of integrated mathematical models. Two historical climate-disease studies of malaria in Africa provide a starting point for further analysis. Early approaches to evaluating the human health risks from global climate change will include simple mapping of disease boundaries and climate factors. Computer-based geographical information system (GIS) technology will assist in the organization and analysis of climate, environment and disease data. Ultimately, complex integrated mathematical models may provide quantitative estimates of risk, but these models have not yet been validated. The collection of geographically organized relevant data through either field work or remote sensing technology will both help validate comprehensive integrated models and enhance our understanding of the associations between climate change and human health.
\end{abstract}

KEY WORDS: Arboviruses - Bioclimatology - Cholera - Dengue - Ecological risk assessment - Exposure ·

GIS - Global warming $\cdot$ Malaria $\cdot$ Modelling $\cdot$ Mosquitos $\cdot$ Remote sensing $\cdot$ Vector-borne diseases

\section{INTRODUCTION}

Initial projections of the vulnerabilities to global climate change have focused on the economic impact of changes in natural resources, such as forests, foodcrops, fisheries, and damage to physical structures. While economic effects undoubtedly would impact upon the health of human populations, international scientists have recently begun exploring more primary effects of global warming on human well-being. Health risks from climate change must not be viewed in isolation from other determinants of human wellbeing, however, and an integrated approach will be required.

Continued accumulation of greenhouse gases in the atmosphere is anticipated to cause global temperature elevations of 1.5 to $4.5^{\circ} \mathrm{C}$ for a doubled atmospheric $\mathrm{CO}_{2}$ concentration (Houghton et al. 1992, IPCC 1994). Altered precipitation patterns, increased weather variability, and thermoexpansion of the oceans are also anticipated from global warming (Tegart \& Sheldon 1992). Subsequent health effects may include an increase in: (1) heat-related mortality and morbidity (Grant 1990, Kalkstein \& Smoyer 1993); (2) infectious diseases, particularly those that are arthropod-borne (Shope 1991, Rogers \& Packer 1993); (3) malnutrition and dehydration from threatened food and water supply (WHO 1990, Parry \& Rosenzweig 1993); and (4) general public health infrastructural damage from weather disasters and sea-level rise, aggravated by climate-related forced human migration (Leaf 1989, Haines \& Fuchs 1991) (Fig. 1). 


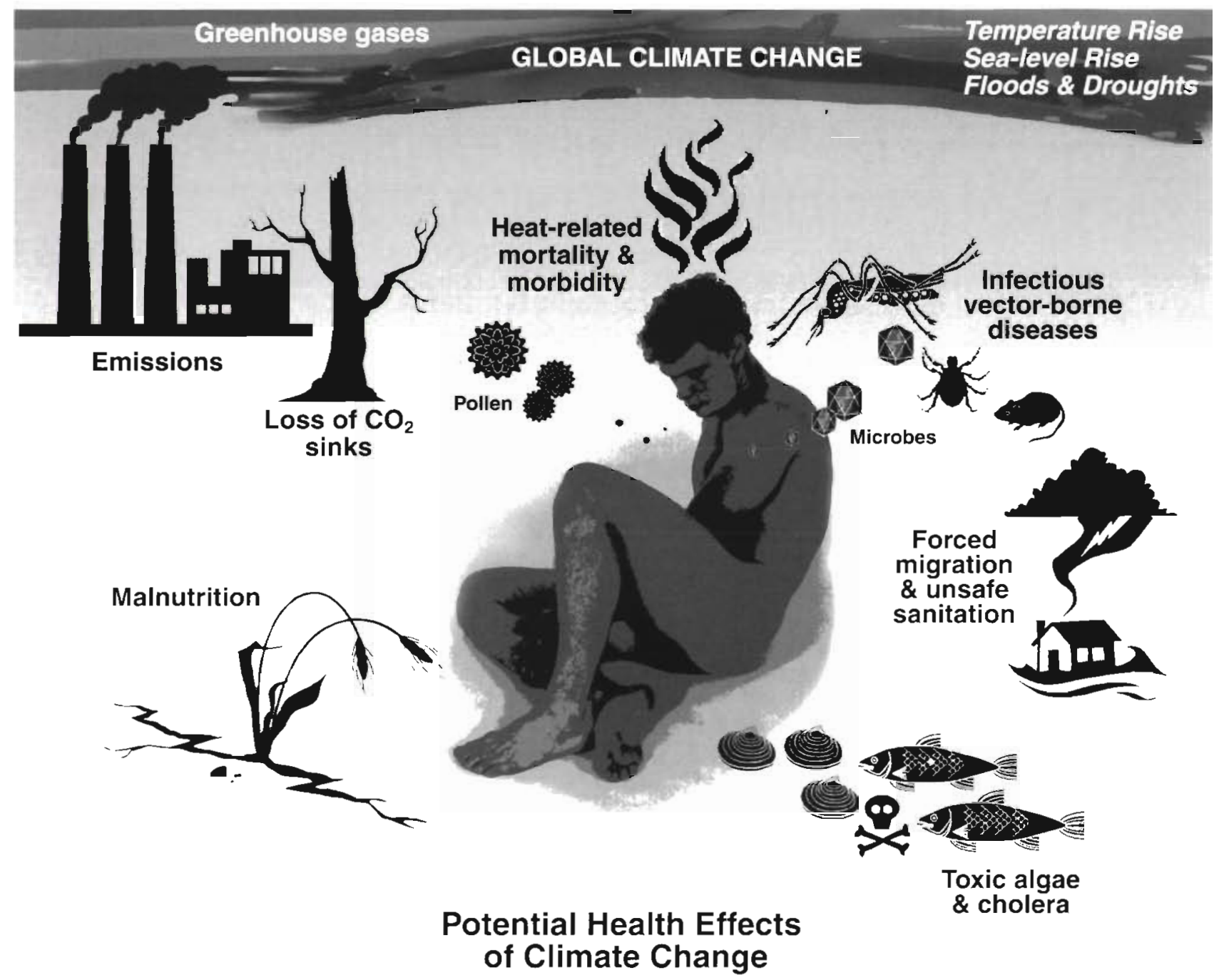

Fig. 1. Illustration of some of the anticipated health effects of global climate change [with permission from Patz JA (in press)]

\section{GENERAL CONSIDERATIONS FOR HEALTH VULNERABILITY ASSESSMENT}

\section{Geographical and time-scale considerations}

The assessment of health vulnerability due to climate change requires the geographical integration of 4 components: climate change itself, subsequent ecological change, human disease activity, and human habitation (Fig. 2) Since each of these components is the outcome of interrelationships among complex determinants, careful and comprehensive organization of data on a geographic basis will be necessary for accurate predictions. Additionally, analysis of human disease systems may be done on a variety of geographic scales, from a small region such as a watershed to an entire country or subcontinent. Such multiple, potentially overlapping analyses requiring the resolution of different layers of data on a variety of geographic scales also necessitates rigorous geographic data organization. The use of computer-aided geographic information systems (GIS) will aid in this geographic organization (detailed below).

Response time of human disease systems to climate change will vary by disease or location. Differences in response time may result from different life spans of intermediate hosts, as well as from differences in interactions and feedback loops at various ecosystem levels. In general, a considerable lag time is anticipated for most human health endpoints. This is because of the inertia of the climate system itself as well as the multiple ecosystem levels which must be altered before human health is affected (with the exception or more direct disease processes, e.g. heat-related mortality). 


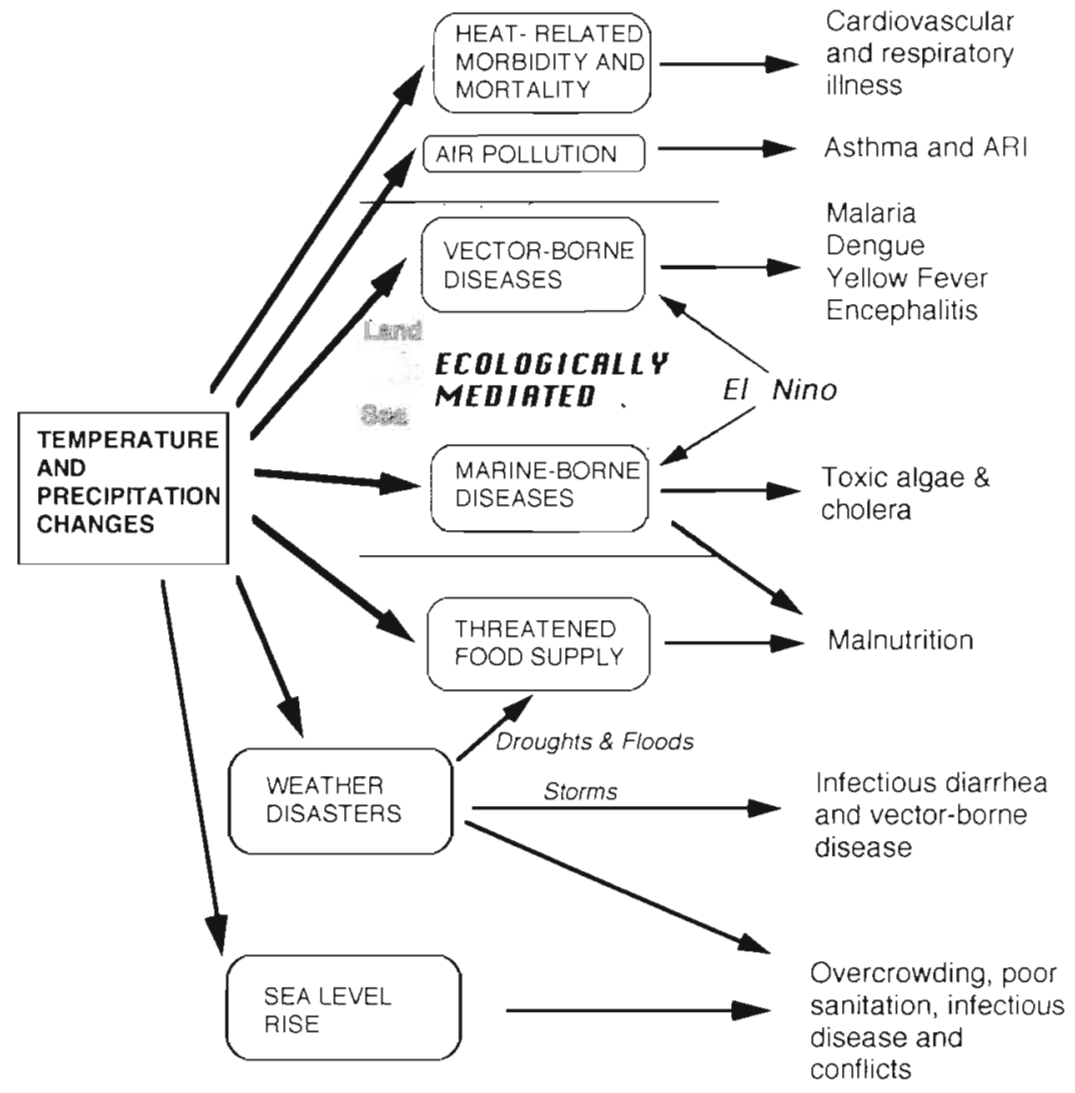

Fig. 2. Causal pathways of public health impacts from climate change

estimations, should still allow for adequate prioritization and relative estimation of risk.

\section{Comparison to conventional risk assessment irameworks}

Conventional human health risk assessment has been developed primarily to evaluate the risk of chemicals in human food and environments. Conventional risk assessment attempts to extrapolate dose-response relationships determined either in a laboratory setting using standardized toxicological assays ${ }^{1}$, or from epidemiologic studies. These approaches may be applicable to some of the hypothesized threats to human health from global climate change, such as ultraviolet radiation induced skin cancers, where epidemiologic data has been essential to determining the doseresponse relationship between exposure and cancer.

This approach will not suffice, however, for many of the health effects anticipated to result from global climate change. Whereas the dose of a conventional hazard is usually expressed as a

\section{Availability of data}

The data requirements for quantitative human health risk assessment related to climate change will be quite large because of the complexity of human disease systems. Problems can be anticipated both in the resolution of the data available as well as the very existence of necessary data. For example, the highest resolution of generally available GCM models approximates $2^{\circ}$ latitude by $4^{\circ}$ longitude, corresponding to an area of nearly $100000 \mathrm{~km}^{2}$ (Dennis Joseph, NCAR, pers. comm.). Analysis of human disease systems may require extrapolation of regional climate predictions to smaller areas, particularly in densely populated areas with highly variant topography.

Where data are not available, simplifying assumptions will be necessary to generate an initial framework for analysis. Such a framework can be used to focus interdisciplinary communication on assessing health risk and to identify priorities for integrated assessment. Surrogate data from neighboring regions or applying assumptions from current trends might be more readily utilized for pressing policy decisions. The use of such assumptions and simplifications, while potentially decreasing the quantitative accuracy of risk magnitude such as concentration, the impact of climate variables such as temperature and rainfall is highly dependent on temporal and geographical characteristics in addition to absolute magnitude. Moreover, the variability, duration, frequency, and timing of climatic events can contribute significantly to subsequent ecological change. The complexity of the dynamic ecological response and feedback to these climate variables necessitates the development of integrated models rather than simple dose-response plots.

Processes that occur at an ecosystem level are not reproducible in a laboratory setting, nor do they follow classical 'dose-response' patterns. Feedback loops and human and non-human adaptive responses are likely to have a significant impact on the ultimate human health vulnerability, since the time scales involved are so long. The ability to predict and model such

\footnotetext{
TThe EPA has recently revised its criteria for cancer risk assessment to be more mechanistic in approaching causal relationships, as opposed to extrapolating merely from highdose studies. Subsequently, more attention is being directed to effects on cellular processes, pharmacokinetics, molecular structure-activity analysis, and low-dose in vivo studies (Stone 1995)
} 
responses and feedback loops is still very limited. A new systems-based method of assessing risk due to interacting environmental factors is required. Nonetheless, analysis of vulnerability may proceed at a far simpler level and still produce useful results.

\section{ECOLOGICALLY BASED HEALTH RISK ASSESSMENT}

Unlike conventional risk assessment, an ecological risk assessment framework allows for evaluation of multiple ecological stress factors and their impacts on a variety of species within an ecosystem. In the context of climate change, an ecological risk assessment may better address nonchemical 'climatic hazards', for which dose-response relationships are less meaningful.

The 1992 U.S. EPA report 'Framework for Ecological Risk Assessment' (Risk Assessment Forum 1992), introduces a framework and terminology which helps distinguish an ecological risk assessment from conventional toxicological paradigms. Three phases define the framework: problem formulation, analysis, and risk characterization (Table 1).

\section{Problem formulation}

Problem formulation requires early consideration of anticipated human health endpoints, and ultimately requires developing conceptual models to help guide analysis. For example, dengue fever outbreaks may depend on sufficient temperatures and moisture avail-

Table 1. Components of an ecologically based health risk assessment as they could apply to malaria. [Adapted from Risk Assessment Forum (1992) U.S. EPA]

\begin{tabular}{|c|c|c|}
\hline Component & & Examples for malaria \\
\hline $\begin{array}{l}\text { Problem formulation } \\
\text { (conceptual model development) }\end{array}$ & $\rightarrow$ & Climate's effect on vectorial capacity \\
\hline Stressor charactenzation & $\rightarrow$ & $\Delta$ water surplus or temperature \\
\hline Endpoint selection & $\rightarrow$ & Man-biting rate of mosquitos \\
\hline \multicolumn{3}{|l|}{ Analysis } \\
\hline Ecosystem characterization & $\rightarrow$ & Vegetative habitat and soil moisture \\
\hline Exposure analysis & $\rightarrow$ & $\begin{array}{l}\text { Water budget and max./min. temps. } \\
\text { extrapolated to a micro-habitat level }\end{array}$ \\
\hline Stressor-response analysis & $\rightarrow$ & $\begin{array}{l}\text { Man-biting rate vs water surplus or } \\
\text { temperature change }\end{array}$ \\
\hline $\begin{array}{l}\text { Risk characterization } \\
\text { Integrated assessment }\end{array}$ & $\rightarrow$ & $\begin{array}{l}\text { Risk defined per site, due to malaria's } \\
\text { varied response by: (1) altitude } \\
\text { (2) human population immune status; } \\
\text { and (3) capacity to adapt, e.g. } \\
\text { mosquito abatement }\end{array}$ \\
\hline
\end{tabular}

ability (influencing mosquito and viral life cycles), human behavior (regarding water storage practices), human migration, demographics and urbanization patterns, and human habitation (i.e. regarding screened windows). These aspects would be incorporated into a conceptual model to guide the analytical stage in addressing the impact of climate change on the key components of this disease system.

Stressor characterization. For climate change, characterizing the 'hazardous exposure' or 'exposure dose' becomes problematic since the magnitude of climate change may not necessarily be the determining factor in human health outcomes. Climate variability, frequency of extreme events, rate of change, or timing of climate events may turn out to be more damaging to an ecosystem than the absolute amount of temperature elevation (Box 1). Thus, stressor characterization will entail predicting both the magnitude and the temporal aspects of climate change.

Intermediate ecosystem changes due to climatic stress may also be conceived of as 'stressors' in a human disease system, since many of the human health impacts from climate change are anticipated to be ecologically mediated, e.g. through insect-vector habitats or marine vegetation. The term stressor, in place of 'hazard,' affords a broader accounting for the processes which can disrupt ecosystems which, in turn, may affect human health.

Endpoint selection and early indicators. Selecting the appropriate human endpoints to evaluate will be based both on empirical observations of diseases that respond to current changes of climate, such as unusual rains or hot spells, as well as on theoretical considerations of conditions that are likely to be altered by climate change (requiring extrapolation from observed climate changes to those predicted in GCM models). These suspected endpoints will require qualitative modelling to help focus analysis and identify contributing ecological factors and intermediate indicators along causal chains (McMichael \& Martens 1995). For example, vegetative land cover which defines insect habitat will affect mosquito populations, which in turn may determine disease outbreaks.

Identification of such intermediate measurable indicators may improve human health risk assessment by aiding in the validation of models. For example, the response of vectorborne infectious diseases to climate change will depend on the existence of intermediate carriers or reservoirs 


\section{Box 1. ENSO events as a possible surrogate for climate change analysis}

(from NOAA's Office of Global Programs)

Some of the anticipated implications for health associated with climate variability may already be within the range of current human experience. The El Nino-Southern Oscillation (ENSO) phenomenon, as an annual driver of short-term climate, may provide insights into the impacts of more long-term increases in climate variability, which are expected to accompany global warming (UNEP \& NCAR 1991, Scripps Institute of Oceanography 1992, Bureau of Meteorology Research Center 1993, Nicholls 1993, Karl et al. 1995).

ENSO is a climate phenomenon which is second only to the annual seasonal cycle in its impacts on regional climate variability (Nicholls 1993). Anomalous fluctuations in precipitation, even to the extent of flood or drought conditions, have been known to result from climate variations influenced by ENSO events which occur approximately every 2 to 8 yr. ENSO most strongly affects countries bordering on the tropical Pacific Ocean, but it is linked through climatic 'teleconnection' to flooding and drought around the world (Glantz et al. 1991). Biological and ecological consequences from year-to-year variability might grant insight into the climate-related threshold effects of global warming on human health. The following examples suggest that for some human health conditions, climate variability may be even more significant than changes in climate mean temperatures.

Extreme heavy rainfalls corresponding to ENSO events have been correlated with outbreaks of mosquito-borne infectious disease (WHO 1995). For example, in temperate southeast Australia, rare but severe epidemics of Murray Valley encephalitis occur after extended periods of rainfall and flooding which correlate with ENSO events (Nicholls 1993). Other vector-borne disease outbreaks tied to climate behavior believed to be influenced by ENSO include Eastern equine encephalitis in the U.S. (WHO 1995), Ross River virus in Australia (Nicholls 1993), and malaria in Sri Lanka, Pakistan, and Argentina (Bouma et al. 1994). In southern Africa, an epidemic of West Nile Fever followed the wet summer of the 1974 ENSO cold phase (Glantz et al. 1991), and persistent El Nino conditions have been cited as one of the contributing factors to the agriculturally devastating drought in countries of the Indian Ocean region in the past several years. Such empirical data stemming from inter-annual ENSOrelated climate variability may aid in health risk assessment of climate change. Such data sets might then be used to validate models such as the one developed by Martens and colleagues to assess the impact of climate change on the incidence of malaria (Martens et al. 1994).

Experience with the human health impacts of ENSO can assist in estimating how populations with varying levels of natural, technical, and social resources would differ in their vulnerability to climate-induced health impacts. Studies of ENSO can be used to refine longer time scale models by helping to separate the influence of climate on human health from the influences of other non-climaterelated parameters. The time scale of ENSO permits the opportunity to implement adjustments in climate forecasting and impacts models, and to learn from mitigation and response strategies. It can introduce a dimension of iterative learning which is not possible on the time scale required to validate models of global warming. ENSO phenomenon may present an opportunity to learn about the ability of human populations to react and adapt to changes in climate on time scales which are of interest to policy makers, and to apply these lessons to long-term planning whose ranges are defined by climate variables. Alterations among the sensitive intermediate species in a human disease system will occur far earlier than changes in human disease incidence or prevalence. By monitoring for such alterations, models may be validated and refined, and opportunities for possible early intervention may become apparent.

\section{Analysis}

The analysis phase takes the conceptual model developed in the problem formulation phase and evaluates the potential responses due to exposure to key stressors. The analysis phase incorporates approaches and terminology that differ from conventional risk assessment. 'Ecosystem characterization' is essential to both exposure and response assessments because of the bi-directional dynamics that often occur between ecosystems (including biotic and abiotic elements) and the climate system. For example, land use changes may affect micro-climate which could alter the effects of climate change on mosquitos, or irrigation of agricultural land may alter a drought's impact on crops. 'Exposure analysis' then attempts to superimpose the spatial and temporal distribution of stressors over relevant ecological components to determine points of contact between stressor and species within an ecosystem. The term 'stressor-response' supplants the concept of conventional dose-response by accommodating the potential multitude of outcomes or processes that can perturb an ecosystem.

Stressor-response analysis. The essential assessment (either quantitative or qualitative) of the impact of stressors on an ecosystem is determined in the stressor-response analysis. From the exposure analysis, described above, the associated magnitude of response is determined for the measurable endpoints identified during the first stage of the assessment. Knowledge of climate-related sensitivity or existence 
of threshold values for each given endpoint is the key to this central step in health risk assessment.

Sensitivity. The term sensitivity as used here refers to the amount a given assessment endpoint is affected by a given amount of change in a climate variable. In conventional terms, this would be analogous to the slope of the 'dose-response' curve in conventional risk assessment. In an ecological risk assessment, because the relation between stressor and assessment endpoint is more complicated, the ultimate 'sensitivity' of a human disease to climate change will often depend on the existence of an organism in the disease system which displays marked alterations in reproductive or other types of behavior in response to changes in climate variables. For example, infectious agents which cycle through cold-blooded insect vectors to complete their development are quite susceptible to subtle climate variations (Dobson \& Carper 1993; see Fig. 3).

Vector-borne microbes, because of their short life span and temperature dependency, can exhibit marked amplification of transmission capacity with increases in temperature. This has been demonstrated for dengue fever, for instance, in Mexico, where investigators found that median temperature during the rainy season was the strongest predictor of dengue infection in the population (Koopman et al. 1991). In support of this field data, laboratory studies have found that the extrinsic incubation period for dengue virus (serotype 2 ) is shortened from the normal 12 day interval at $30^{\circ} \mathrm{C}$ to 7 days at 32 to $25^{\circ} \mathrm{C}$ (Watts et al. 1987) Shortening the incubation period by 5 days could potentially triple the transmission rate of the disease (Koopman et al. 1991). Thus, the sensitivity of dengue fever as a human disease system to climate change is suggested by this potential tripling in viral transmission rate in response to temperature increases within the range predicted by the IPCC assessment.

Thresholds. A concept related to sensitivity is that of 'threshold'. In conventional risk assessment, a 'threshold' refers to a sudden change in the slope of the doseresponse curve. For example, human sensitivity to temperature extremes varies on a physiologic basis. Heat-related mortality occurs at different temperatures depending on the latitude and typical tempera tures for that area. For example, whereas Montreal shows an upswing in heat-related mortality at $29^{\circ} \mathrm{C}$, Dallas does not show a similar increase until $39^{\circ} \mathrm{C}$ (Kalkstein \& Smoyer 1993). Thus, Montreal may be said to have a temperature threshold at $29^{\circ} \mathrm{C}$, while Dallas exhibits a threshold at the higher temperature of $39^{\circ} \mathrm{C}$. This variation necessitates separate analyses for each region that attempts a vulnerability assessment of heat-mortality.

When considering ecological risk assessment, the threshold may be better conceptualized as a point of

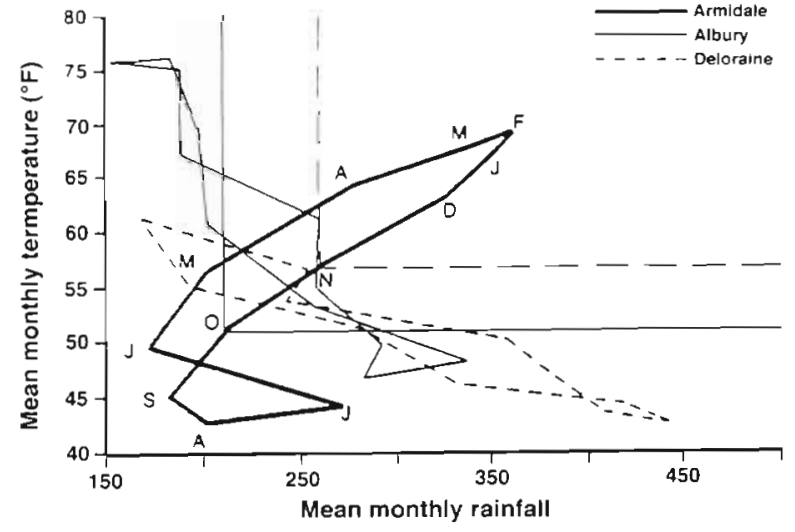

Fig. 3. Example of a bioclimatograph for a nematode species, $H$. contortus. There appears to be a distinct bioclimatic 'envelope' in which a species thrives. Also, notice the variation between 3 geographic locations [from Dobson \& Carper (1993) with permission]

non-linear behavior of an ecosystem endpoint. Such non-linearity may be the expression of climate conditions which limit or prevent adequate reproduction of an intermediate species. Conversely, non-linearity in the behavior of an ecosystem may relate to some critical reproductive or population parameter above which exponentially increasing transmission can occur. Thresholds may occur temporally, as changing climate within a given geographic area alters the behavior of an already-present human disease. They may also manifest geographically, as changing climate conditions allow migration of human disease into a previously unaffected area.

This climate dependency translates into constrained geographic ranges within which human disease transmission can occur. A central concern related to the health effects of climate change is that global warming and climate change may extend the range of viability for infectious diseases, leading to the spread of disease into new geographic areas inhabited by more immunologically susceptible human populations. Such geographic change in the behavior of human diseases is likely to take place around ecotones, or border areas of transition between different ecosystems. As an example, the malaria parasite cannot develop inside its mosquito host at temperatures below $16^{\circ} \mathrm{C}$ (Gilles 1993). This constitutes a temperature threshold that may translate into altitude or other geographic borders for malaria in a given region. In Eastern Africa, many of the major population centers are situated at altitudes above the endemic areas of malaria. Should temperature increases in eastern Africa cross this $16^{\circ} \mathrm{C}$ threshold, malaria would be anticipated to spread into present ecotones between the low and higher altitude ecosystems. Thus, simultaneous understanding of the climate and geographic boundaries of human diseases 
is essential to predicting regional vulnerability to climate change (Box 2).

\section{Risk characterization}

Risk characterization results from the integration of stressor characterization and stressor-response analysis. To the extent possible, human adaptive responses should be integrated into the risk characterization. While a given disease system may be particularly sensitive to the effects of climate change based on biological or physiological characteristics, the ultimate vulnerability of humans to that disease may be considerably lessened by adaptive responses. For example, projections of temperature increase in the temperate latitudes would render large areas in temperate regions susceptible to a resurgence of malaria (Jetten \& Takken 1994, Martens et al. 1994, Matsuoka \& Kai 1995). It is likely, however, that widespread increases in malaria incidence would be mitigated by pesticide use and other technological adaptations. Thus, while malaria as a disease system may be sensitive to climate change based on its biology, the population in developed countries would not likely be vulnerable because of economic and technological resources. Characterization of risk both in the presence and absence of adaptive responses may be useful in evaluating the cost-benefit characteristics of different adaptive responses. Such analyses will be greatly facilitated by integrated mathematical modelling (Martens 1996).

Uncertainty analysis must accompany the overall integrated assessment. Uncertainty will be present at all levels of the risk assessment. During the conceptual model phase, incorrect assumptions may prove difficult to resolve. The inevitability of incomplete data must be addressed throughout the analysis, and errors in measurement and sampling will need to be transparent throughout the assessment process. Finally, the natural variability, or 'stochasticity' within climate and ecological systems must be adequately represented (Risk Assessment Forum 1992). Communication regarding uncertainties must occur between scientists and policy makers early on in the process to insure that the results of the risk assessment are accurately represented to constituencies at risk.

\section{DEVELOPING METHODS FOR CLIMATE CHANGE/HEALTH ASSESSMENT}

There is a need for a 2-tiered approach to the risk assessment of climate change, including the gathering of empirical climate/health data which serves as the foundation for the second phase, scenario-based math- ematical modelling. Empirical analysis uses historical climate and disease data to attempt to establish a causal link between climate and disease activity. Scenario-based mathematical modelling, on the other hand, uses climate data derived from computer models and disease models derived from both field and laboratory data to make predictions about disease activity. While validated quantitative models are still in the development stages, qualitative evaluation may be useful for guiding public policy and for directing future research efforts. The improvement of data resources will allow better vulnerability assessment in the future as both climate change and human disease models become more refined and validated; this requires capacity building for improved surveillance and monitoring to detect changes.

\section{Empirical climate change analysis}

Meteorological data. A variety of meteorological approaches can be used in the historical analysis of the impact of climate on health: human comfort indices, synoptic climatological classifications, water budget analyses and energy budget analyses (WHO 1995). The method to be utilized will depend on the health outcome of interest. For example, the synoptic classification scheme, which characterizes distinct air masses, affords greater predictive value than the use of single climate variables such as temperature and relative humidity in modelling heat mortality in temperate regions (Kalkstein 1991). On the other hand, a water budget approach, which compares supply, or precipitation, with climatic demands for water, such as through evapotranspiration, may be more useful for other diseases (Mather 1993). Insect vectors which include an aquatic stage in their life cycle will lend themselves to a water budget model to study climate/vector-borne disease dynamics. For example, in Burkina Faso, where onchocerciasis is endemic, a water budget model was implemented to accurately predict black fly populations which depend on adequate stream flow rates for their development (Mills 1995).

Evaluating gradual changes in climate and disease variables will necessitate the use of time series analysis, which involves the examination of multiple comparisons over a sequence of time (UNITAR 1994). Data gathered over a long continuous period and at short time intervals provide the best data for time series analysis, but are often difficult to obtain. 'Change' analysis, which involves pair-wise analysis between 2 dates or, in the case of remotely sensed satellite data, 2 images, may be useful in the assessment of climaterelated ecological change. 


\section{Box 2. Climate and geographic distribution of malaria: methodologies from an African regional workshop}

During March 1995, representatives from ministries of health and environment from 8 African countries (Botswana, Kenya, Malawi, Mozambique, Tanzania, Uganda, Zambia and Zimbabwe) attended the regional Health Vulnerabilities to Climate Change Workshop of the U.S. Country Studies Program. Workshop participants identified malaria and malnutrition as the top priority climatesensitive diseases for the southeastern African region. Two studies from Rwanda and Zimbabwe, respectively, were used as examples of how malaria incidence could potentially change in response to climate factors.

Temperature and humidity are the most important factors for malaria transmission and the extrinsic incubation period of the pathogen shortens dramatically at temperatures above $20^{\circ} \mathrm{C}$ (Gilles 1993). Climate factors which increase the breeding activity of Anopheles mosquitos are probably the most important cause of epidemic outbreaks since small increases in transmission can produce major epidemics (Gilles 1993)

The relationship of malaria incidence to local climatic change has been observed in Rwanda. In 1987, extension of malaria into higher altitudes resulted after record high temperatures and rainfall (Loevinsohn 1994). Monthly malaria incidence in high altitude regions was exponentially affected by changes in minimum temperature. In low altitude zones, rainfall and mean temperature were the most significant climate factors (Fig. 4). The study concluded that areas near the current limits of malaria distribution would potentially be most vulnerable to climatic changes. Based on such observations, a relatively small increase in winter (minimum) temperature might facilitate the spread of malaria into large urban highland populations that are currently malaria-free, such as Nairobi in Kenya and Harare in Zimbabwe.

To no surprise, this study also found that the greatest increase in malaria incidence occurred in groups with the least acquired immunity to malaria: children aged $<2$ yr and populations inhabiting high-altitude regions. Therefore, changes in disease incidence among children in areas adjacent to, but not within, endemic regions could be an early indication of the spread of malaria.

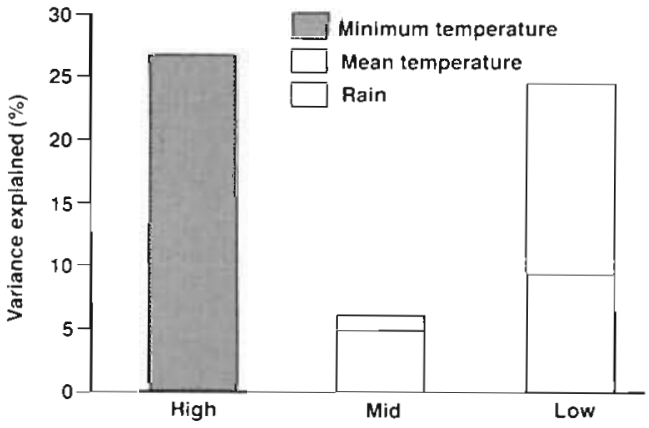

Fig. 4. Proportion of variance of log monthly malaria incidence accounted for by climatic variables in best-fitting equations of 3 altitude zones (with permission from Loevinsohn (1993)]
A Zimbabwe study showed malaria prevalence to strongly vary inversely with altitude (Taylor \& Mutambu 1986). In Zimbabwe, malaria ranged from being hyperendemic at altitudes below $600 \mathrm{~m}$ to being absent on the central highlands above $1200 \mathrm{~m}$ (Figs. 5 \& 6). For the altitudes between these extremes, 'malaria is unstable and epidemic in nature' (Taylor \& Mutambu 1986). Since most of the population of Zimbabwe lives at these intermediate to high altitudes, based on the results of the Rwandan study, a subtle shift in temperatures might be expected to significantly affect the public health of the region.

One way to begin to assess vulnerability to changing incidence of malaria due to climate change is to map regions suitable for Anopheles mosquitos and the Plasmodium pathogen under new climate scenarios. The first step

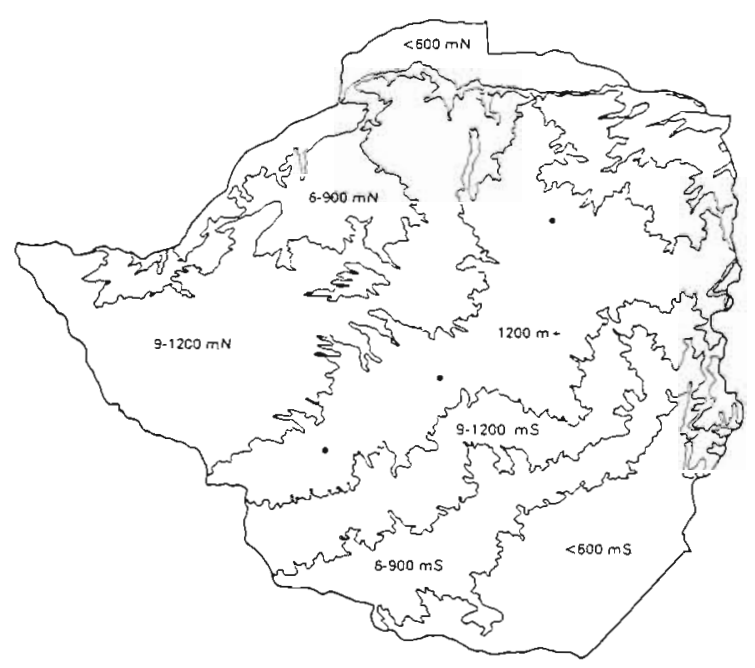

Fig. 5. Altitude classification of Zimbabwe (with permission from Taylor \& Mutambu (1986)|

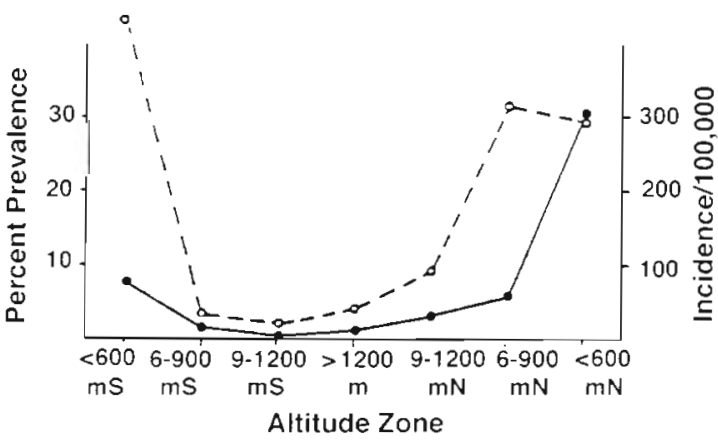

Fig. 6. Average annual prevalence (•) and incidence/ 100000 population (0) of malaria by altitude zone for the years 1969-1981 and 1972-1981, respectively |with permission from Taylor \& Mutambu (1986)] 
would involve mapping the current malaria endemic zones and obtaining averaged weather data for that corresponding area (Country Studies protocol is to attempt this for 30 yr of climate data, preferably from 1951 to 1980). By superimposing climate predictions based either on General Circulation Model (GCM) scenarios or by arbitrary incremental changes in temperature or precipitation, the potential new endemic area might be 'redrawn.' One important step towards this analysis has already been completed by Unganai (1996; this issue). Predicted minimum temperatures for a 3 month period have been mapped under a doubled $\mathrm{CO}_{2}$ scenario using GCMs of the Canadian Climate Center and the Geophysical Fluid Dynamics Laboratory (see Unganai 1996).
Though not comprehensive, this methodological approach provides an important step towards assessing the health vulnerabilities due to climate change. Subsequent analysis of overall regional risks might involve including outputs from this mapping exercise within a larger integrated framework. For example, while increased rainfall at low altitudes may markedly increase the incidence of malaria, it would simultaneously reduce the risk of malnutrition due to drought. Also, with greater water availability, the risk of diarrheal diseases diminishes. The need for advances in integrated assessment is obvious; however, this should not discourage the pursuit of more directed analysis of significant priority outcomes such as malaria.
Epidemiological data. Epidemiology is the study of determinants and distribution of diseases within human populations. Fitted models can help predict health risk, given similar etiologic conditions, by statistical analysis of empirical data. Dynamic mathematical models have also been utilized in the early prediction of human outbreaks for some disease 'systems' such as vector-borne diseases, which may be most sensitive to climate. Transmission rate is expressed in terms of 'vectorial capacity', $C$, which provides the daily rate of potentially infectious contacts between vector and host. Ultimately, the rate of potentially infective bites can then be determined:

$$
C=\frac{m a^{2} p^{n}}{-\log _{e} p}
$$

where $m$ is the number of vectors per host, $a$ is the daily number of blood feedings per vector, $p$ is the vector's daily survival probability, and $n$ is the number of days between the vector's ingestion of an infected blood meal and the point at which it becomes capable of producing infectious disease (the 'extrinsic incubation period') (Reiter 1988). This example illustrates the predictive advantages that a mathematical model may provide so that public health personnel might be better prepared to anticipate disease epidemics emerging from climate-induced changes in any one of the independent variables. These variables may also serve as measurable endpoints, or bioindicators, within an ecological risk assessment.

The above equation applies only to 'virgin' populations where the immune barrier to infection is absent. Consideration of the immune status of the human population is essential to achieve quantitative predictions of the spread of infectious vector-borne diseases. Pesticide and antibiotic resistance in some mosquito and protozoan populations, respectively, need to also be integrated into the assessment (Centers for Disease Control and Prevention 1994).

\section{Integrated modelling}

A specific disease model, such as the vectorial capacity equation illustrated above for vector-borne diseases could be embedded within a more integrated systems approach to better reflect the complexity of the broader relationships between climate, intermediate ecosystem changes, human health, and human adaptive capacity. Such analysis can account for interactions or feedback processes and can simulate dynamic changes over time (Rotmans et al. 1994). The article on the TARGETS model in this issue of Climate Research (Martens 1996) further details the application of systems analysis for health risk assessment. However, as the author points out, uncertainties accumulate with each model linkage, and large aggregated models may begin to misrepresent reality at a certain point.

Most human diseases have more determinants than just vector populations or climate variables (Table 2). Many of the primary determinants of human health (adequate food, clean water and secure shelter) are related to the outcomes of sectors such as agriculture, water resources and fisheries. In populations suffering malnutrition problems, the effect of climate change on agricultural production may have a greater adverse effect on human health than any given disease. Therefore, it is important to integrate these relevant systems into the human health assessment. Mathematical models are also necessary because predicted climate changes lie outside the range of past observations. A number of integrated models are currently under development for such diseases as dengue and malaria (Focks et al. 1993, Jetten \& Takken 1994, Martens et al. 1994, Matsuoka \& Kai 1995).

Understanding of human disease and the ability to model disease rates in the absence of climate change is still relatively undeveloped. While public health scientists agree that socioeconomic factors play a significant role in health and susceptibility to disease, just how to 
Table 2. Factors in disease emergence. [From the Institute of Medicine (1992) Emerging infections: microbial threats to health in the United States. National Academy Press, Washington, DCl

\begin{tabular}{|ll|}
\hline Categories & Specific examples \\
\hline Societal events & $\begin{array}{l}\text { Economic impoverishment; war or civil conflict; } \\
\text { population growth and migration; urban decay }\end{array}$ \\
& $\begin{array}{l}\text { New medical devices; organ or tissue trans- } \\
\text { plantation; drugs causing immunosuppression; } \\
\text { widespread use of antibiotics }\end{array}$ \\
Food production & $\begin{array}{l}\text { Globalization of food supplies; changes in food } \\
\text { processing and packaging }\end{array}$ \\
Human behavior & $\begin{array}{l}\text { Sexual behavior; drug use; travel; diet; outdoor } \\
\text { recreation; use of child care facilities }\end{array}$ \\
$\begin{array}{l}\text { Environmental } \\
\text { changes }\end{array}$ & $\begin{array}{l}\text { Deforestation/reforestation; changes in water } \\
\text { ecosystems; flood/drought; famine }\end{array}$ \\
$\begin{array}{l}\text { Public health } \\
\text { infrastructure }\end{array}$ & $\begin{array}{l}\text { Curtailment or reduction in prevention programs; } \\
\text { inadequate communicable disease surveillance; lack } \\
\text { of trained personnel (epidemiologists, laboratory } \\
\text { scientists, vector and rodent control specialists) }\end{array}$ \\
$\begin{array}{l}\text { Changes in virulence and toxin production; } \\
\text { development of drug resistance; microbes as chronic } \\
\text { cofactors in diseases }\end{array}$ \\
$\begin{array}{l}\text { Microbial } \\
\text { adaptation } \\
\text { and change }\end{array}$
\end{tabular}

reveal early influences of climate change that would not yet have resulted in human disease, since insect and protozoan species react more quickly to changes in their environment. While such surveillance may not provide a short-term answer to questions of vulnerability, it may provide the quantitative long-term data through which more accurate models can emerge. To the extent that early indicators or 'precursors' of disease are monitored, increased opportunities for even earlier preventive measures might result.

Geographic information systems (GIS). Because much of the analysis of human health vulnerability will require the superimposition of data on disease incidence, vector populations, demographics, and climate with linkage to specific geographic locations, a geographic method of organizing and storing this data will be very useful. A number of software programs provide GIS technology. The use of GIS systems for predicting disease incidence is new and still under development. It should be stressed that GIS systems will

quantify socioeconomic factors for use in disease prediction has not yet been determined. Moreover, even if there were adequate ways to express socioeconomic factors quantitatively, there is a general paucity of data, both for socioeconomic and disease variables. Without geographically organized data to input, even the best models will have difficulty making accurate predictions.

\section{Capacity building for improved assessment and early response}

Surveillance and monitoring. Attention needs to be directed at detecting changes in climate-driven diseases in sensitive geographic regions bordering endemic zones. Establishing sentinel diagnostic centers located in such regions should be given consideration. Such detection will not only provide an early warning for possibly more pervasive changes in disease behavior and allow for intervention, it will also enrich our knowledge on climate dependency of diseases and facilitate the creation of better predictive models.

Another surveillance approach which may be easier to perform and also provide earlier warning for vectorborne disease spread is the monitoring of habitat changes which influence vector species populations and infectivity. Monitoring their populations may not provide higher quality outputs than the quality of the data which is entered into them; therefore attention needs to be paid to the proper collection and organization of the data before computerized analysis is initiated.

GIS technology can assist in: (1) data collection; (2) data management; (3) data querying; (4) modelling; (5) decision-making (Glass et al. 1993). Data sources can come either from standard record-keeping activities or from more targeted survey data. Remotely sensed data would be especially useful in areas where data on population distribution, land use patterns, or transportation patterns are lacking (Glass et al, 1993). For current problems, GIS can better enable identification of areas in need of intervention and help assess impacts of such measures. In the long-term, any geographical trends resulting from climate change might be recognized sooner.

Remote sensing. Remote sensing (or remote satellite imaging) can be useful in the analysis of diseases when their distribution or epidemiology depends on climate and landscape features which determine habitats. Vector-borne diseases are particularly influenced by spatial and temporal fluctuations in vector populations. Vegetation partly determines vector populations, since plant composition in a region reflects the aggregate effect of temperature, precipitation and humidity (Washino \& Wood 1994). Satellite generated vegetative index or habitat maps have been used to identify areas 
receptive to dengue fever and Lyme disease in the USA, schistosomiasis in the Philippines, and malaria in rice growing regions (Washino \& Wood 1994).

Studies from Africa demonstrate the potential utility of applying remote sensing technology by predicting both insect-vector abundance and pathogen activity within vectors. In one study in sub-Saharan Africa, remote sensing was applied to predict the regional risk of trypanosomiasis or African sleeping sickness which is carried by tsetse flies (Rogers \& Randolph 1991). The study showed a correlation between tsetse fly population density and vegetative characteristics identified from satellite images.

In another study addressing Rift Valley Fever, satellite imagery of vegetation in south-central Kenya was used to indicate levels of recent rainfall (Linthincum et al. 1987). The authors found that in areas with a lush vegetation index by satellite pictures, virus activity was detectable in the corresponding mosquito populations. These 2 studies demonstrate the benefit of applying remote sensing technology to identify geographic habitat characteristics which may predict subsequent human disease. Areas of high risk can also be identified to help in achieving earlier and better focused control measures.

In combination, GIS and remote sensing technology may serve as a useful analytical tool. GIS allows for overlaying of maps with multiple components, some of which are easily acquired by satellite. For example, Landsat images can discern standing pools of water. and locate human settlements (Epstein et al. 1993). When superimposed over maps of climatological, entomological and epidemiological data, enhanced integrated analysis may be possible.

\section{CONCLUSION}

The health effects expected from climate change will most likely stem from multiple environmental processes. Impacts may occur through changes in agriculture, natural resources such as fresh water or fisheries, or via multiple species within ecosystems, such as rodents or insect-vectors. Excepting heat waves or weather disasters, most of these ecologically mediated threats will involve complex ecological feedbacks in non-linear systems. A systems-based, ecological risk assessment is therefore in order.

Integrated mathematical modelling will be required for simulating such complex interactions, as well as for accounting for varying capacities to adapt to climatic changes across different populations. Accepting inevitable uncertainty, modelling can help in the analysis of interactions between climate change, subsequent ecological change, human disease activity, and human habitation. Yet, more emphasis needs to be placed on the process of modelling, rather than on the numerical outputs which are generated. That is, in the process of model building, a deeper understanding may emerge as researchers begin to identify key gaps in data and as answers are sought from a growing number of disciplines.

Natural, economic and demographic factors must be integrated into the health risk assessment. To the extent that data in key areas is missing, more simplified and focused analysis should be encouraged in parallel with integrated modelling. Many of the consequences of climate change will be manifested geographically, primarily by shifts in disease distribution or by localized alterations in the supply of healthrelevant resources. The mapping of endemic areas of disease, as discussed in Box 2, could help identify sensitive locales on which to focus monitoring and surveillance efforts which could identify shifting disease trends early on. Computer-based GIS and remote sensing technology can assist in the organization and analysis of this type of data and can enhance our understanding of the associations between climate change and human health.

Considering the magnitude of the potential public health impacts from climate change, interdisciplinary links for promoting an integrated assessment need to be advocated (Table 3). Yet, for a challenge as complex and potentially large as global climate change, discussion must extend beyond scientists from diverse fields to include policy makers at all levels of the assessment. In an area with much uncertainty, such cooperation will enhance initial problem formulation and, ultimately, the risk communication necessary for making informed decisions affecting the health of the public.

Table 3. Steps for improved health risk assessment of climate change

(1) Conduct scientific studies of pertinent diseases with readily available databases to build up foundation of empirical information

(2) Integrate mathematical modeling techniques with empirical studies to help establish a comprehensive integrated assessment of the health impacts from climate change scenarios

(3) Encourage multidisciplined and international collaboration to maximize expertise and promote long-term funding in the integrated assessment process

(4) Establish climate/health relvant monitoring and surveillance initiatives in vulnerable regions

(5) Communicate research findings to inform the public and to help guide policy and future scientific research 
Acknowledgements. The authors were enabled to undertake this project thanks to funding support from the U.S. EPA's Climate Change Division, cooperative agreement number 823143010, as well as funding from the U.S. Country Studies Program. We are extremely grateful to Macol Stewart and Claudia Nierenberg from NOAA's Office of Global Programs for their preparation of the section on ENSO events. We also thank Dr Margaret McDonnell for her excellent assistance towards preparing the Health Vulnerabilities Presentation for the African Regional Workshop of the U.S. Country Studies Program. We extend thanks to Prof. Anthony McMichael at the London School of Hygiene and Tropical Medicine, and Dr Joan Aron from Johns Hopkins University, for their invaluable comments and revisions during the development of the manuscript.

\section{LITERATURE CITED}

Bouma MJ, Sondorp HE, van der Kaay HJ (1994) Climate change and periodic epidemic malaria. Lancet 343:1440

Bureau of Meteorology Research Center (1993) Climate change and the El Nino-Southern Oscillation: report of the workshop on climate change and the El Niño-Southern Oscillation. Bureau of Meteorology Research Center, Report 36

Centers for Disease Control and Prevention (1994) Addressing emerging infectious disease threats: a prevention strategy for the United States. U.S. Dept. of Health and Human Services, Public Health Service, Atlanta

Dobson A, Carper R (1993) Biodiversity. Lancet 342 (October): $1096-1099$

Epstein PR, Rogers DJ, Slooff R (1993) Satellite imaging and vector-borne disease. Lancet 341:1404-1406

Focks DA, Haile DG, Daniels E, Mount GA (1993) Dynamic life table model for Aedes aegypti (L.) (Diptera: Culicidae). J Med Entomol 30:1.003-101.7

Gilles HM (1993) Epidemiology of malaria. In: Gilles HM Warrell DA (eds) Bruce-Chwatt's essential malariology. Edward Arnold Div. of Hodder \& Stoughton, London

Glantz MH, Katz RW, Nicholls N (1991) Teleconnections linking worldwide climate anomalies. Cambridge University Press, Cambridge

Glass GE, Aron JL, Ellis JH, Yoon SS (1993) Applications of GIS technology to disease control. The Johns Hopkins University, Baltimore

Grant LD (1990) Respiratory effects associated with global climate change. In: White JC (ed) Global atmospheric change and public health. Elsevier, New York

Haines A, Fuchs C (1991) Potential impacts on health of atmospheric change. J Pub Health Med 13(2):69-80

Houghton JT, Callander BA, Varney SK (eds) (1992) Climate change 1992: the supplementary report to the IPCC scientific assessment. Cambridge University Press, Cambridge

IPCC (1994) Radiative forcing of climate change, the 1994 report of the scientific assessment working group of IPCC: summary for policy makers. Oxford University Press, Oxford

Jetten TH, Takken $W$ (1994) Impact of climate change on malaria vectors. Clim Change 18:10-12

Kalkstein LS (1991) A new approach to evaluate the impact of climate upon human mortality. Environ Health Perspect 96: 145-150

Kalkstein LS, Smoyer KE (1993) The impact of climate change on human health: some international implications. Experientia 49:469-479
Karl TR, Knight RW, Easterling DR, Quayle RG (1995) Trends in the U.S. climate during the twentieth century. Consequences $1: 3-12$

Koopman JS, Prevots DR, Marin MAV, Dantes HG, Aquino MLZ, Longini IMJ, Amor JS (1991) Determinants and predictors of dengue infection in Mexico. Am J Epidemiol 133: $1168-1178$

Leaf A (1989) Potential health effects of global climatic and environmental changes. N Engl J Med 321:1577-1583

Linthincum KJ, Bailey CL, Davies FG, Tucker CJ (1987) Detection of Rift Valley Fever viral activity in Kenya by satellite remote sensing imagery. Science 235:1656-1659

Loevinsohn $M$ (1994) Climatic warming and increased malaria incidence in Rwanda. Lancet 343:714-718

Martens WJM (1996) Global atmospheric change and human health: an integrated modelling approach. Clim Res $6: 107-112$

Martens WJM, Rotmans J, Niessen LW (1994) Climate change and malaria risk: an integrated modeling approach. RIVM, GLOBO Report Series 3, Report 461-502-003, Bilthoven

Mather JR (1993) Workbook in applied climatology. Publ Climatol 46:2-109

Matsuoka Y, Kai K (1995) An estimation of climatic change effects on malaria. J Global Environ Engineering 1:43-57

McMichael AJ, Martens WJM (1995) The health impacts of global climate change: grappling with scenarios, predictive models, and multiple uncertainties. Ecosystem Health $1(1): 23-33$

Mills DM (1995) A climatic water budget approach to black fly population dynamics. Publ Climatol 95

Nicholls N (1993) El Niño-southern oscillation and vectorborne disease. Lancet 342:1284-1285

Parry ML, Rosenzweig C (1993) Food supply and the risk of hunger. Lancet 342:1345-1347

Patz JA (in press) Global climate change and public health. In: Shahi G, Levy B, Kjellstrom T, Lawrence R, Binger A (eds) International perspectives in environment, development and health: toward a sustainable world. Springer Publications, New York

Reiter P (1988) Weather, vector biology, and arboviral recrudescence. In: Monath TP (ed) The arboviruses: epidemiology and ecology. CRC Press, Boca Raton, p 245-255

Risk Assessment Forum (1992) Framework for ecological risk assessment. U.S. Environmental Protection Agency, EPA/630/R-92-001, Washington, DC

Rogers DJ, Randolph SE (1991) Mortality rates and population density of tsetse flies correlated with satellite imagery. Nature 351:739-741

Rogers DL, Packer MJ (1993) Vector-borne diseases, models, and global change. Lancet 342:1282-1284

Rotmans J et al (1994) Global change and sustainable development: the TARGETS approach. RIVM, Report 4.61502004, Bilthoven

Scripps Institution of Oceanography (1992) Simulated global warming and model ENSO cycles. Scripps Institution of Oceanography, Ref 9404, San Diego

Shope RE (1991) Global climate change and infectious diseases. Environ Health Perspectives 96:171-174

Stone R (1995) A molecular approach to cancer risk (news \& comment). Science 268:356-357

Taylor P, Mutambu SL (1986) A review of the malaria situation in Zimbabwe with special reference to the period 1972-1981 Trans Roy Soc Trop Med Hyg 80:12-19

Tegart WJM, Sheldon GW (eds) (1992) Climate change 1992 the supplementary report to the IPCC impacts assessment Australian Government Publishing Service, Canberra

United Nations Environment Program (UNEP) and the 
National Center for Atmospheric Research (1991) ENSO and climate change. Bangkok

United Nations Institute for Training and Research (1994) Change and time series analysis. UNITAR, Geneva

Washino RK, Wood BL (1994) Application of remote sensing to arthropod vector surveillance and control. Am J Trop Med Hyg 50(Suppl 6):134-144
Watts DM, Burke DS, Harrison BA (1987) Effect of temperature on the vector efficiency of Aedes aegypti for dengue 2 virus. Am J Trop Med Hyg 36:143-152

WHO (1990) Potential health effects of climatic change. World Health Organization, Geneva

WHO (1995) Climate and health in a changing world. World Health Organization, Geneva 